\title{
Research progress of natural plant products inducing apoptosis of tumor stem cells
}

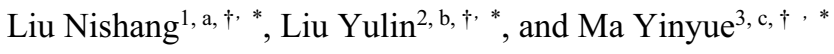 \\ ${ }^{1}$ School of Biological science, Sichuan Agriculture University, Xi'an, Shanxi 710038, China; \\ ${ }^{2}$ School of Biology, Hunan University, Changsha, Hunan 410000, China. \\ ${ }^{3}$ School of Life sciences, South China Agricultural University, Guangzhou, Guangdong 510642, China/School of Agriculture and Food \\ Science, University College Dublin, Belfield, Dublin 4, D04 V1W8, Ireland; \\ ${ }^{\dagger}$ These authors contributed equally.
}

\begin{abstract}
Compared to general cancer cells, cancer stem cells are stronger and more difficult to be killed by drugs. Therefore, traditional treatment methods have low efficacy according to cancer stem cells. In essence, one of the main causes of cancer survival, proliferation, transfer, and recurrence is the presence of cancer stem cells. In recent years, a large number of studies have demonstrated that natural plant products have powerful in killing cancer cells and have various structures, which possess low toxicity, multi-target regulation advantages in inducing cancer stem cells to apoptosis. Therefore, this provides new ideas for developing anti-cancer drugs by inducing cancer stem cell apoptosis by natural plant products. In this article, we will introduce the apoptotic mechanism of tumor stem cells, the importance of eliminating tumor stem cells, and the benefits of natural plant products in cancer treatment. In addition, this paper reviewed nine categories of chemicals in natural plant products that induce apoptosis of tumor stem cells and their mechanisms of action. We also summarized the mechanism of natural plant products inducing apoptosis of tumor stem cells. This review provides an important reference for the personalized treatment of cancer.
\end{abstract}

\section{Introduction}

In 1967, Bergagel first put forward the Cancer Stem Cell (CSC) theory. It pointed out that when a subclone population of cells was on the verge of elimination, less than $5 \%$ of the cells would survive and obtain more malignant biological phenotypes, such as tumorigenesis and metastasis ability, self-renewal ability and drug resistance [1]. CSC is the conventional name of a small proportion of these cells. It is becoming clearer that the vast majority of tumors have specialized stem cells [2], and CSC also has three classical signaling pathways Wnt, Shh, and Notch in adult stem cells. Chemotherapy, radiotherapy, or targeted therapy can hardly completely identify and eliminate CSC, which leads to the tumor shrinking or even disappearing at first. Still, it will relapse soon, leading to the death of patients [3]. Studies have found that CSC can escape the killing of drugs through dormancy, produce drug resistance through abnormal expression of ABCG2 and other molecules [4], or achieve the goal of drug resistance through the activation of Wnt, Shh, and Notch signaling pathways in cells. In conclusion, cancer eradication requires killing cancer stem cells.

Modern medicine combined with traditional Chinese medicine can combine the advantages of Chinese and Western medicine, give full play to the strengths of each family, and run through the whole process of cancer treatment. The low content of active components, difficulty in extraction and separation, and poor chemical stability of natural plant products lead to fewer clinical trials. However, their active components can act on different stages of carcinogenesis, have low toxicity characteristics, and act on multiple targets of a pathway. Stem cell apoptosis is a very complex process involving many signaling molecules, and the failure of inducing cancer stem cell apoptosis is one of the main obstacles in the treatment of cancer. Plant natural products can induce stem cell apoptosis through various targets, and these apoptotic pathways are regulated by many pro-apoptotic or anti-apoptotic molecules [5]. Studies have found that curcumin [6] and other chemicals in natural plant products can induce stem cell apoptosis. They can also effectively prevent precancerous lesions by inhibiting the signaling pathway of carcinogenesis. In addition, studies

*Corresponding author Email:a20156095@stu.sicau.edu.cn,bly1428@hnu.edu cjoycema0623@126.com/yinyue.ma@ucdconnect.ie 
have shown that the effective chemical components extracted from Chinese herbal medicine plants play a synergistic role in combination with immunotherapy. When used together with some traditional chemotherapy drugs, these natural plant products can effectively reduce the cytotoxicity of traditional chemotherapy drugs in the treatment. The use of natural plant products for anti-tumor is one of the research directions of new anti-tumor drugs.

This paper first emphasizes the importance of eliminating cancer stem cells and the benefits of natural plant products in cancer treatment. Then, from the perspective of inducing apoptosis of cancer stem cells by natural plant products, different natural plant products are divided into nine categories according to chemical classification. They cover 20 substances, and the mechanism of inducing apoptosis of different cancer stem cells by these substances in their ways is clarified. It also provides an essential comprehensive reference for inducing apoptosis of cancer stem cells and provides a clear reference for personalized treatment of cancer. The various natural product extracts appearing in this article and their tumor stem cell markers are shown in Table 1.

Table 1 Cancer stem cell markers of various natural products

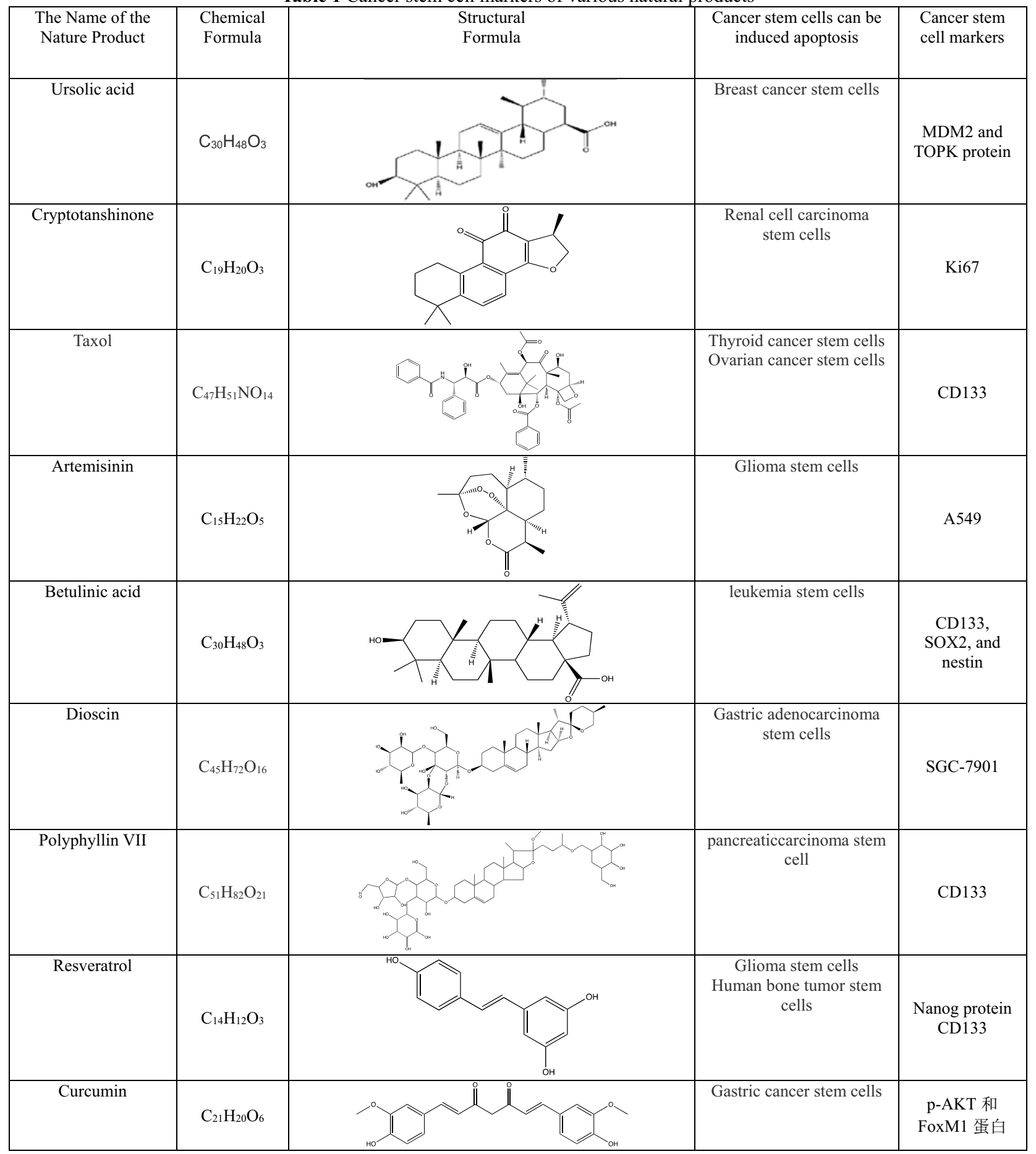




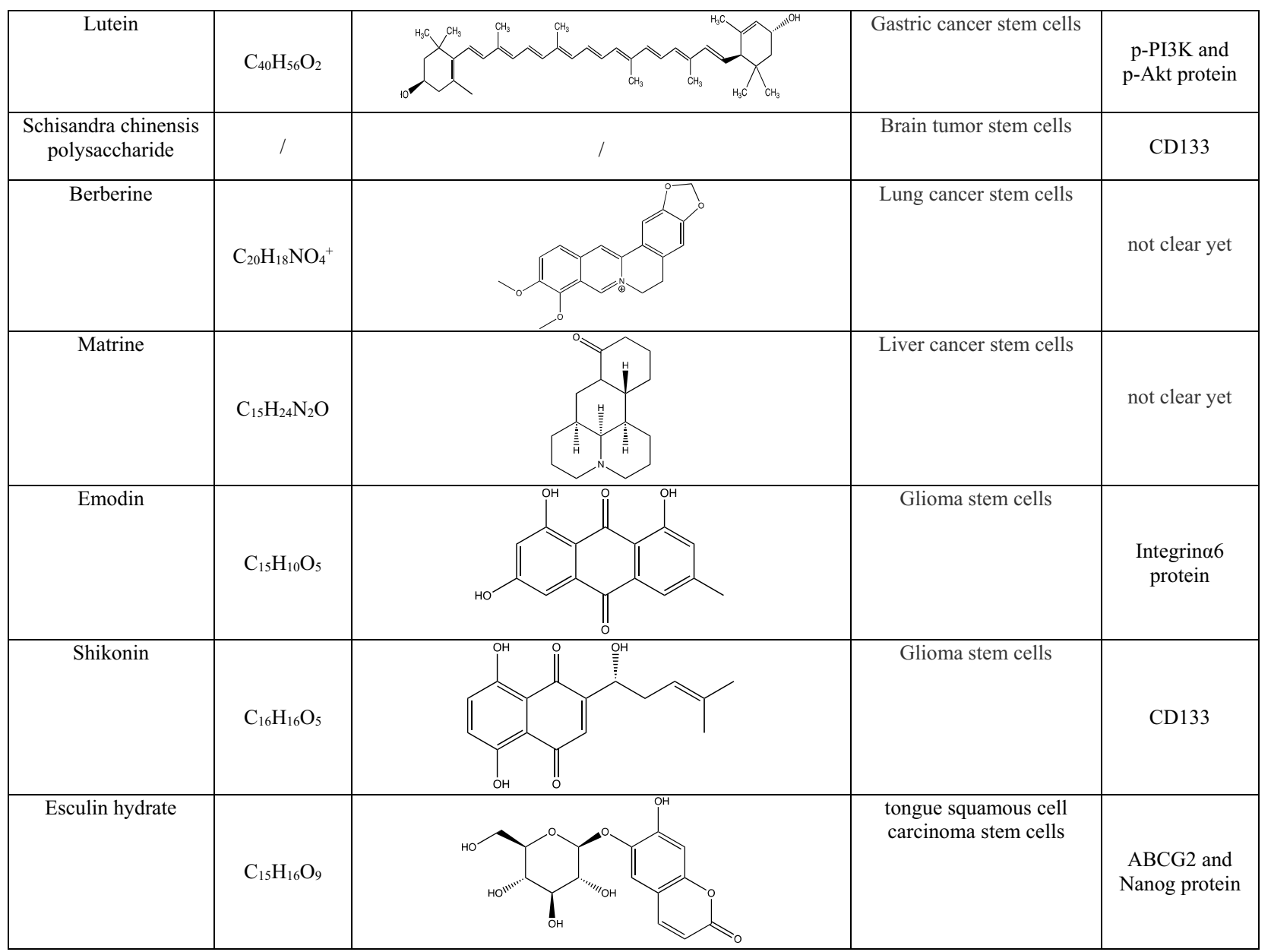

\section{Contents}

\subsection{Plant natural products induce apoptosis of cancer stem cells}

\subsubsection{Terpenoids and apoptosis of cancer stem cells}

\subsubsection{Ursolic acid}

Ursolic acid(UA or 3 3 -hydroxyurs-12-en-28-oic acid) is a triterpenoid widely distributed in the plant kingdom. Ursolic acid can be isolated from different plants, such as rosemary officinalis, Origanum majorana L., lavandula angustifolia mill., thymus mongolicus, and leaves of some organic herbs, and some parts of fruits, like apple peel, flower, and berry [7]. In addition, the results of some recent researches showed that ursolic acid could inhibit the proliferation of CSCs and induce the apoptosis of CSCs. Interestingly, ursolic acid also can promote autophagy and apoptosis of breast cancer stem cells by inhibiting the activation of Akt [8].

\subsubsection{Cryptotanshinone}

Cryptotanshinone is a fat-soluble component extracted from Salvia miltiorrhiza Bge, which is easily soluble in organic solvents such as dimethyl sulfoxide and methanol and slightly soluble in water [9]. What's more, Cryptotanshinone has many pharmacological activities such as anti-tumor, anti-inflammator,y and anti-oxidation in vivo and in vitro experiments [10]. Based on some researches, Feng min et al. found that cryptotanshinone at the concentration of $5 \mathrm{mg} / \mathrm{L}$ can induce the apoptosis of renal cancer stem cells by inhibiting the expression of $\mathrm{Ki} 67$ and $\mathrm{Bcl}-2$ and promoting the expression of p-caspase-3 [11].

\subsubsection{Taxol}

Taxol is a natural secondary metabolite isolated from the bark of Taxus baccata Linn. While, through clinical verification, paclitaxel has a certain anti-tumor effect, especially for ovarian cancer, uterine cancer, and breast cancer [12]. Then, in the research of Yang Jiuyi and her group, they have used several steps to get their conclusions. First, the cells treated with paclitaxel for 72 $\mathrm{h}$ and transfected with TGM2 siRNA for $24 \mathrm{~h}$ were collected, and the total RNA was extracted by the Trizol lysis method. Then, MTS was used to detect the effect of inhibition of TGM2 expression on paclitaxel sensitivity. Western blot was used to detect the protein expression of CD133 - and CD133 + cells and flow cytometry was used to detect cell apoptosis. Based on these methods, it was found that the expression of E-cadherin was significantly increased. In contrast, the expression of vimentin and messenger RNA (mRNA) was significantly decreased in the combination group [13]. What's more, Ganlei and her group through culture and passage of ovarian cancer stem like cells, detection of ovarian cancer stem cell-related marker gene Oct-4 by qRT-PCR, detection of cell proliferation by tetramethylazo salt colorimetry, flow cytometry were used to detect apoptosis and other test methods and procedures found 
that the expression of E-cadherin was significantly increased. In contrast, the expression of vimentin and mRNA was significantly decreased in the combination group. As noted, it is speculated that the combination of apatinib and docetaxel may interfere with the epithelial mesenchymal transition process of ovarian cancer stem cells and then promote the apoptosis of ovarian cancer stem cells [14].

\subsubsection{Artemisinin}

Artemisinin is a bioactivator derived from the Compositae plant Artemisia annua Linn. [15]. In addition to antimalarial activities, artemisinin, and its derivatives, dihydroartemisinin and artesunate, also have anti-tumor effects. Cao et al. found that dihydroartemisinin could inhibit the proliferation of glioma stem cells by inhibiting the phosphorylation expression of Akt and activating caspase-3, respectively, and arrest glioma stem cells in the G1 phase of the cell cycle, jointly achieving the purpose of apoptosis of glioma stem cells [16].

\subsubsection{Betulinic acid}

Betulinic acid (BA) is a five-ring lupine triterpene, which is widely found in nature. It was first isolated from the bark of evergreen plants of the Rhamnaceae family growing in Eastern Africa. It up-regulates Bax, inhibits Bcl-2 and survivin genes, causes the loss of mitochondrial transmembrane potential, thereby activating caspase 9 and activating downstream caspase $3 / 7$, which leads to the apoptosis of leukemia stem cells [17].

\subsubsection{Saponins and cancer stem cell apoptosis}

\subsubsection{Dioscin}

Dioscin is a kind of steroidal saponins commonly found in plant rhizomes, especially in the rhizomes of Dioscoreaceae plants, such as Dioscorea nigra, Dioscorea zingiberensis, etc. [18]. Hu et al. found that after acting on human gastric adenocarcinoma stem cells, the antiproliferative effect of dioscin on human gastric carcinoma SGC-7901 cells was confirmed by 3-(4, 5-dimethylthiahiazo-zyl)-2, 5-dip-henytetrazolium bromide and flow cytometry assays. In addition, increased mRNA expression of p53 was also found in dioscin-treated SGC-7901 cells, and the activation of caspase- 3 and - 8 was also observed. Therefore, Dioscin promotes the apoptosis of gastric adenocarcinoma stem cells by up-regulating Bax and down-regulating Bcl-2, thereby activating the mitochondrial pathway [19].

\subsubsection{Polyphyllin VII}

Polyphyllin VII belongs to steroidal saponins, which has been proved to have an anticancer effect by inhibiting cell proliferation and inducing cell apoptosis [20]. Polyphyllin VII can inhibit the proliferation, migration, and invasion of pancreatic cancer Miapaca-2 cells, induce apoptosis, inhibit the proliferation of pancreatic cancer stem cells, and the expression of stem cell marker CD133 in vitro. However, how Polyphyllin VII regulates the expression of pancreatic cancer stem cells' CD133 is still unclear and needs further research [21].

\subsubsection{Polyphenols and cancer stem cell apoptosis}

\subsubsection{Resveratrol}

Resveratrol is a natural small-scale polyphenol compound with the chemical formula of C14H12O3, which can be extracted from various plants such as grapes, peanuts, mulberry, and so on. It has been verified that resveratrol has a significant therapeutic effect on a variety of tumors [22]. In addition, resveratrol can also induce apoptosis of tumor stem cells, including human osteoma stem cells and glioma stem cells. Wang and his group [23] found that resveratrol could inhibit the expression of Nanog by activating the P53 gene, and activate the autophagy activity of glioma stem cells, inhibit their proliferation capacity, and promote their apoptosis.

\subsubsection{Curcumin}

Curcumin is a type of natural polyphenol compound with a diketone structure extracted from Zingiberaceae and Araceae's rhizomes. The molecular formula of curcumin is $\mathrm{C} 21 \mathrm{H} 20 \mathrm{O}$. Curcumin has the effects of oxidation resistance, anti-inflammation, and metabolic regulation. Curcumin can regulate the proliferation and differentiation of cells through cell energy metabolism. It can also play a physiological role by regulating signaling pathways such as apoptosis through pattern recognition receptors [24]. Studies [25] have revealed that curcumin can inhibit the expression of p-AKT and FoxM1 in gastric cancer stem cells by blocking the Akt/FoxM1 signaling pathway, thereby inhibiting the proliferation of gastric cancer stem cells and promoting their apoptosis.

\subsubsection{Carotenoids and apoptosis of cancer stem cells}

Lutein is a kind of natural plant pigment mainly extracted from Tagetes erecta L, which has anti-oxidation functions and improves human immunity [26]. Interestingly, Lutein can also be used as an anti-tumor drug. Du et al. found that lutein can inhibit the proliferation of various tumor cells and promote apoptosis. Also, it can reduce the expression of p-pi3k and p-Akt protein by down regulating Akt / FoxM1 signaling pathway so that the protein molecules play the role of apoptosis induction and inhibition of proliferation, and make gastric cancer stem cells apoptosis

\subsubsection{Polysaccharides and apoptosis of cancer stem cells}

Schisandra chinensis polysaccharide is extracted from the water extract of Chinese herbal medicine Schisandra chinensis. In addition, many studies have shown that Schisandra polysaccharide has very high pharmacological effects, such as liver protection, immune regulation, antioxidant, antitussive, and anti-tumor effects [28]. For instants, Ding et al. found that Schisandra polysaccharide has an inhibitory effect on the growth of brain tumor stem cells through experiments. Schisandra polysaccharide can increase the pro apoptotic factors of brain tumor stem cells by regulating the ratio of $\mathrm{Bax} / \mathrm{Bcl}-2$, increasing the activated Caspase-3, and decreasing the tumor stem cell 
marker CD133. It indicates that Schisandra polysaccharide can induce the apoptosis of brain tumor stem cells [29].

\subsection{6 alkaloids and cancer stem cell apoptosis}

\subsubsection{Berberine}

Berberine, also known as berberine, is an alkaloid compound separated from Rhizoma Coptidis with the chemical formula of $\mathrm{C} 20 \mathrm{H} 18 \mathrm{NO} 4$. Berberine has the effects of anti-diarrhea, anti-bacteria, anti-inflammation, and so on. In recent years, studies have found that berberine has an inhibitory effect on various tumors such as breast cancer, pancreatic cancer, lung cancer, and other tumors with little toxicity and side effects [30]. Moreover, Sun et al. have revealed that berberine can inhibit the growth of lung cancer stem cells, possibly inhibit the proliferation of lung cancer stem cells and promote their apoptosis by regulating the Hedegehog signaling pathway [31].

\subsubsection{Matrine}

Matrine is an alkaloid extracted from the plant and fruit of Radix Sophorae Flavescentis of Leguminosae with an organic solvent, and its molecular formula is $\mathrm{C} 15 \mathrm{H} 24 \mathrm{~N} 2 \mathrm{O}$. Matrine has a wide range of biological activity. It can protect the heart, blood vessels, liver, lung, and other parts with anti-ulcer, sedative, and hypnotic effects. Meanwhile, it can also play a role in immune regulation and the anti-tumor effect [32]. Studies have shown that matrine inhibits the proliferation of liver cancer stem cells and reduces its ability to attack and metastasis [33]. However, its mechanism of inducing apoptosis of liver cancer stem cells is still unclear.

\subsubsection{Quinones and cancer stem cells apoptosis}

Emodin is a quinone compound extracted from the dried rhizome of Rhizoma Polygoni Cuspidati, and the chemical formula is $\mathrm{C} 15 \mathrm{H} 10 \mathrm{O} 5$. Studies have found that emodin can play an anti-tumor role by inhibiting the proliferation and migration of tumor cells, inducing apoptosis and other ways, and having a therapeutic effect on liver cancer, lung cancer, colon cancer, breast cancer, and pancreatic cancer [34]. Through experiments, Kim et al. found that emodin could inhibit the STAT3, Notch-1, $\beta$ - catenin pathway of glioma stem cells, block the self-renewal of glioma stem cells, inhibit the expression of EGFR, and reduce the expression of Integrina6 protein, thereby inducing the apoptosis of glioma stem cells [35].

\subsubsection{Natural naphthoquinones and cancer stem cell apoptosis}

Shikonin exists in the roots of Lithosperraum erythrorhizon Sieb. et Zucc., Arnebia euchroma (Royle) Johnst and other plants. It is a naphthoquinone compound extracted from the traditional Chinese medicine Comfrey. Its chemical formula is $\mathrm{C} 16 \mathrm{H} 16 \mathrm{O} 5$ [36]. Shikonin can significantly inhibit the dryness of glioma stem cells (GSCs) [37], it can affect the expression of P21 [38], affect the cell cycle, and inhibit the PI3K/Akt signaling pathway [39].

\subsubsection{Coumarin glucosides and cancer stem cell apoptosis}

Esculin hydrate is the main active ingredient of traditional Chinese medicine Qinpi. It is the Oleaceae plant Fraxinusrhynchophylla Hance, Fraxinuschinensis Roxb. , Fraxinusszaboana Lingelsh or Fraxinusstylosa Lingelsh. The chemical formula is C15H16O9 [40], including 4 kinds of dried branch bark or dried bark extract. Qinpi A can promote the apoptosis of tongue squamous cell carcinoma stem cells (ALDH br cells) by inhibiting the activation of the Rac1/LIMK1/cofilin signaling pathway.

\subsection{Conclusion and outlook}

At present, conventional treatment cannot significantly improve the survival rate of cancer patients, indicating that it is time to adopt a new personalized treatment plan. In summary, the natural products of Chinese herbal medicine have made great achievements in inducing apoptosis of cancer stem cells. As an important part of complementary and alternative medicine, natural products of Chinese herbal medicines have been paid more and more attention to their toxicity and safety. Still, reasonable treatment and optimized application can avoid this problem. The active ingredients and derivatives of natural plant products have the effects of inhibiting proliferation, inducing cell cycle arrest, inhibiting invasion and migration, inducing apoptosis, anti-angiogenesis, and improving immunity. However, since the current number of studies is still relatively small, there is still a need to further expand the research sample size in the future. In addition, we also found that the same active ingredient can act on different signaling pathways. Therefore, this multi-target, multi-level, low-toxicity treatment method that induces tumor stem cell apoptosis will bring new directions for tumor treatment or adjuvant therapy shortly.

\section{Reference}

1. BERGSAGEL DE. The chronic leukemias: a review of disease manifestations and the aims of therapy[J]. Can Med Assoc J,1967,96(25):1615-1620

2. Gao Xiaomei, Zhang Kaili, Yu Xinxin \& Qin Lunxiu. (2016). The role and status of stem cells in solid tumor metastasis. Fudan Journal (Medical Edition) (01), 81-85. doi:CNKI:SUN:SHYK. 0.2016-01-018.

3. Colak, S., \& Medema, J. P. (2014). Cancer stem cells--important players in tumor therapy resistance. The FEBS journal, 281(21), 4779-4791. https://doi.org/10.1111/febs.13023

4. COJOC M, MABERT K, MUDERS MH, et al. A role for cancer stem cells in therapy resistance: cellular and molecular mechanism[J]. Semin Cancer Biol,2015,31:16-27 
5. Xiukun Lin,,Pingxiang Xu,,Keming Zhang,... \& Huanli Xu.(2015).Antitumor effects of traditional Chinese medicine targeting the cellular apoptotic pathway. Drug Design, Development and Therapy(default),. doi:10.2147/DDDT.S80902.

6. Afrin, R., Arumugam, S., Rahman, A., Wahed, M. I., Karuppagounder, V., Harima, M., Suzuki, H., Miyashita, S., Suzuki, K., Yoneyama, H., Ueno, K., \& Watanabe, K. (2017). Curcumin ameliorates liver damage and progression of NASH in NASH-HCC mouse model possibly by modulating HMGB1-NF- $\mathrm{BB}$ translocation. International immunopharmacology, 44, 174-182. https://doi.org/10.1016/j.intimp.2017.01.016

7. Yang Ling \& Su Xiulan. (2019). The effect of ursolic acid on tumors and cancer stem cells. Chinese Journal of Histochemistry and Cytochemistry,374-380.doi:10.16705/j.cnki.1004-18 50.2019.04.013.

8. Zhang, X., Song, X., Yin, S., Zhao, C., Fan, L., \& $\mathrm{Hu}, \mathrm{H}$. (2016). p21 induction plays a dual role in anti-cancer activity of ursolic acid. Experimental biology and medicine (Maywood, N.J.), 241(5), 501-508. https://doi.org/10.1177/1535370215616195

9. Zeng Jin, Zhang Zhirong, Miao Ping, Shen Shujiao, Jiang Jian \& Qiu Furong. (2015). Research progress on the pharmacological effects of cryptotanshinone. Chinese patent medicine (06), 1309-1313. doi:CNKI:SUN:ZCYA. 06-033

10. Kang Jichuan, Jin Rui, Wen Tingchi, He Jin \& Lei Bangxing. (2011). Review and prospects of taxol production by endophytic fungi. Acta Mycologica Sinica (02), 168-179. doi: 10.13346/j .mycosystema.2011.02.001.

11. Feng Min \& Jia Minghua. (2016). The effect of cryptotanshinone on the proliferation and apoptosis of renal cancer stem cells. Chinese Tissue Engineering Research (01), 49-54. doi:CNKI:SUN:XDKF.0.2016-01-011 .

12. Wang, W., Li, X., Han, X. Z., Meng, F. B., Wang, Z. X., Zhai, Y. Q., \& Zhou, D. S. (2015). Transglutaminase-2 is Involved in Cell Apoptosis of Osteosarcoma Cell Line U2OS Under Hypoxia Condition. Cell biochemistry and biophysics, 72(1), 283-288. https://doi.org/10.1007/s12013-014-0451-1

13. Yang Jiuyi, Jia Siyue, Wu Lingqiao, Zhang Caifen, Gong Jianping \& Kong Dandan. (2015). The effect of paclitaxel combined with cisplatin on the proliferation, migration and invasion of thyroid cancer cell SW579. China Pharmacy (34), 4782-4785. doi:CNKI:SUN:ZGYA.0.2015-34-012.

14. Gan Lei, Huang Yake, Huang Yan \& Xie Rongkai. (2020). The effect of apatinib combined with docetaxel on ovarian cancer stem cells and its mechanism of action. Medical review (23), 4769-4775. doi: .

15. Xu Zangzang, Xiao Linfan, Wen Shun \& Mou Lingli. (2020). Research progress on the effects of artemisinin and its derivatives on cancer stem cells. China Wild Plant Resources (08), 50-53. doi:CNKI:SUN: ZYSZ.0.2020-08-010.

16. Cao L, Duanmu W S, Yin Y, et al. Dihydroartemisinin exhibits anti - glioma stem cell activity through inhibiting $\mathrm{p}-\mathrm{AKT}$ and ac- tivating caspase - 3[J]. Pharmazie, 2014, 69( 10): 752 - 758.

17. Leong KH, Mahdzir MA, Din MF, Awang K, Tanaka Y, Kulkeaw K, Ishitani T, Sugiyama D. Induction of intrinsic apoptosis in leukaemia stem cells and in vivo zebrafish model by betulonic acid isolated from Walsura pinnata Hassk (Meliaceae). Phytomedicine. 2017 Mar 15;26:11-21. doi: 10.1016/j.phymed.2016.12.018. Epub 2017 Jan 9. PMID: 28257660.

18. Chen Yaqin, Cao Yongjun. Research progress in modern pharmacology of dioscin [J]. Modern Journal of Integrated Traditional Chinese and Western Medicine, 2019, 28(23): 2613-2617.

19. $\mathrm{Hu} \mathrm{M}, \mathrm{Xu} \mathrm{L}$, Yin L, et al.Cytotoxicity of dioscin in human gastric carci- noma cells through death receptor and mitochondrial pathways[J]. J Appl Toxicol, 2013, 33( 8) : 712-722.

20. Esmaeili M, Farimani MM, Kiaei M.Anticancer effect of calycopterin via PI3K / Akt and MAPK signaling path- ways, ROS - mediated pathway and mitochondrial dys- function in hepatoblastoma cancer (HepG2) cells[J]. Mol Cell Biochem, 2014, 397( $1 / 2): 17-31$.

21. Hu Shishang, Zhou Yuanyuan, Wu Zihao, et al. Study on the effect of Chonglou saponins VII on pancreatic cancer cells [J]. Journal of Hubei Medical College, 2020, 39(6): 544-550.

22. Peng Lihua. (2019). Research on the inhibitory effect of resveratrol on osteosarcoma stem cells and related mechanisms (Doctoral dissertation, Chongqing Medical University).

23. Wang, L. , Long, L. , Wang, W. , \& Liang, Z. . (2015). Resveratrol, a potential radiation sensitizer for glioma stem cells both invitro and invivo sciencedirect. Journal of Pharmacological Sciences, 129(4), 216-225.

24. Ruan Dong, Wang Yibing, Jiang Shouqun \& Zheng Chuntian. (). The biological activity of curcumin and its molecular mechanism for regulating the barrier function of animal intestinal mucosa. Journal of Animal doi:10.3969/j.issn.1006-267x .2021.04.000.

25. He Dongli. (2016). Curcumin regulates the proliferation and apoptosis of gastric cancer stem cells through the ATK/FoxM1 signaling pathway. Chinese Tissue Engineering Research (32), 4731-4737.

26. Chen Cheng, Cheng Xi, Huang Conglin, Luo Chang \& Liu Yanfen. (2016). Research progress on extraction methods and functions of lutein. Hebei Forestry Science and Technology (03), 71-75. doi:10.16449/j.cnki .issn1002-3356.2016.03.026. 
27. Du Ruiling. (2019). Lutein regulates the proliferation and apoptosis of gastric cancer stem cells through the PI3K/AKT signaling pathway. Chinese Tissue Engineering Research (29), 4593-4598. doi:CNKI:SUN:XDKF.0.2019-29- 003.

28. Yu Haoran, Tian Zhenkun, Gao Xiang, Chen Xiaoyu \& Wu Lun. (2018). Progress in the pharmacological effects of Schisandra chinensis polysaccharides. Chemical Engineer (07), 64-67. doi: 10.16247/j.cnki.23-1171/tq. 20180764.

29. Ding Zhendong, Zhang Yuying, Zhang Yu, Zhong Yue, Wen Na, Yu Hongquan \& Qi Ling. (2018). Schisandra polysaccharide induces apoptosis and growth inhibition of brain tumor stem cells. Journal of Jilin University (Medical Edition) (02),305-309+465.doi:10.13481/j.1671-587x.201802 18.

30. Yang Xianhao, Zhang Lijin, Luo Mengjun, Luo Shuai, Gong Yuyuan \& Chen Tao. Research progress in the anti-tumor mechanism of berberine. Chinese Journal of Chinese Materia Medica. doi:10.19540/j.cnki.cjcmm.20210209.601.

31. Sun Yanzhen, Li Zhen \& Yuan Zheng. (2017). The effect of berberine on the proliferation and apoptosis of lung cancer stem cells and its mechanism. Chinese Tissue Engineering Research (09), 1313-1318. doi:CNKI:SUN:XDKF .0.2017-09-003.

32. Zhang Mingfa \& Shen Yaqin. (2020). Progress in the pharmacokinetics of matrine. Drug Evaluation Research (12), 2571-2578. doi:CNKI:SUN:YWPJ.0.2020-12-040.

33. Wang Yong, Liu Yahui, Jiang Jianshuai \& Cui Hanbin. (2013). The inhibitory effect of matrine on the proliferation and invasion and metastasis of liver cancer stem cells. Chinese Journal of Experimental Surgery (01), 61-63. doi:CNKI:SUN:ZHSY .0.2013-01-029.
34. Li Donghui, Wang Linyan, Wu Hongwei, Zhang Shujuan, Zhang Yugui \& Li Yuefeng. New progress in the research of emodin's pharmacological effects. Chinese Journal of Traditional Chinese Medicine (),

35. Park, M. J., Kim, J. Y. , Lee, J. S. , Jung, J. , Imm, I. , \& Lee, J. Y. . (2014). Emodin suppresses maintenance of stemness by augmenting proteosomal degradation of egfr/egfrviii in glioma stem cells. Stem Cells \& Development.

36. Li Zeyun, Chen Zhiqiang \& Lin Ruiting. (2019). Research progress of traditional Chinese medicine intervention in cancer stem cells. Chinese Folk Therapy (10), 104-105. doi:10.19621/j.cnki.11-3555/r.2019.1057.

37. Xiong Sihui, Wan Sha, Tan Li, Lu Xiaolu, Shi Mengying, Liu Li \& Xu Haibo. (2017). Current status of research on the regulation of cancer stem cells by traditional Chinese medicine. World Traditional Chinese Medicine (10), 2535-2538+2542. doi:.

38. Wang, F., Mayca Pozo, F., Tian, D., Geng, X., Yao, X., Zhang, Y., \& Tang, J. (2020). Shikonin Inhibits Cancer Through P21 Upregulation and Apoptosis Induction. Frontiers in pharmacology, 11, 861. https://doi.org/10.3389/fphar.2020.00861

39. Zhang, F. L., Wang, P., Liu, Y. H., Liu, L. B., Liu, X. B., Li, Z., \& Xue, Y. X. (2013). Topoisomerase I inhibitors, shikonin and topotecan, inhibit growth and induce apoptosis of glioma cells and glioma stem cells. PloS one, 8(11), e81815.https://doi.org/10.1371/journal.pone.008181 5.

40. Li Gan \& Xia Dajing. (2019). The effect of Qinpi A on the proliferation and oxidative stress of ECV304 cells damaged by AGEs. Natural Products Research and Development (08), 1439-1446. doi: 10.16333/j.1001 -6880.2019.8.020. 